\title{
Biopolitical Surveillance Vs COVID-19 in Authoritarian and Democratic States and Its Possible Post-Pandemic Implications
}

\author{
Minas Samatas* \\ Emeritus Professor of Sociology, University of Crete, Greece
}

*Corresponding Author: Minas Samatas, Emeritus Professor of Sociology, University of Crete, Greece

\begin{abstract}
The urgency of coping with the COVID-19 pandemic has enforced even democratic governments to adopt restrictions and surveillance applications working in authoritarian regimes. In this presentation we use the term "biopolitical surveillance" (BS) for both functions: the supervision of the restrictions to limit the spreading of COVID-19 and the surveillance of personal life with collection and processing of sensitive health, communication and contact data. BS is understood as an emergency biopolitics, which is however going to be continued in the post-pandemic "risk society" and "post-democracy", likewise with the continuing 9/11 Patriot Act." According to international organizations and NGO's, urgent institutional safeguards and citizens awareness are required to avoid the post-pandemic BS harm of privacy, freedoms and democracy.
\end{abstract}

Keywords: biopolitical-surveillance, COVID-19, 11/9, freedoms, democracy

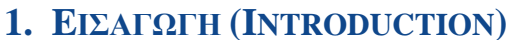

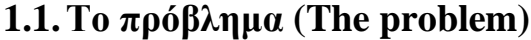

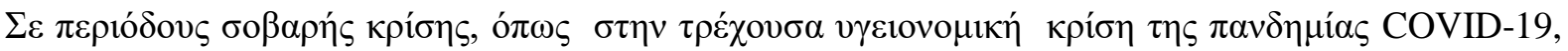

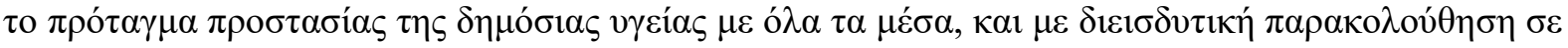

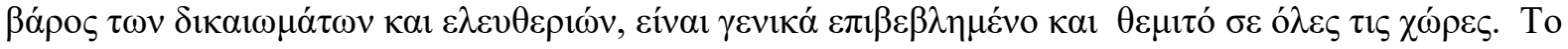

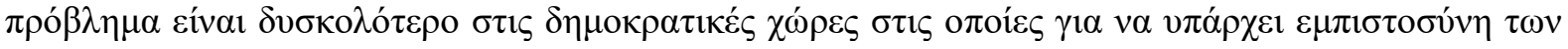

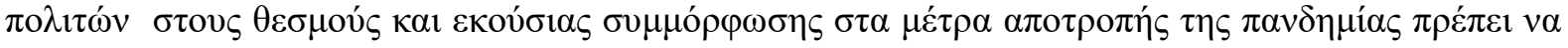

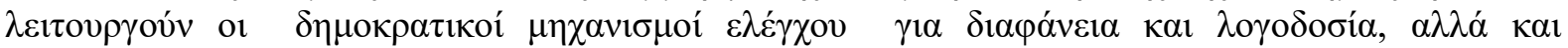

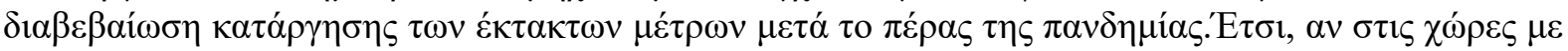

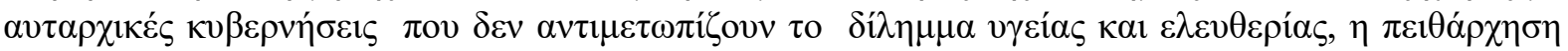

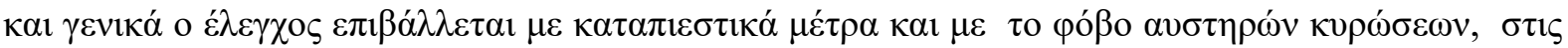

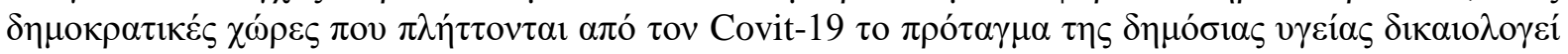

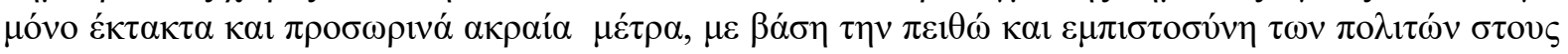

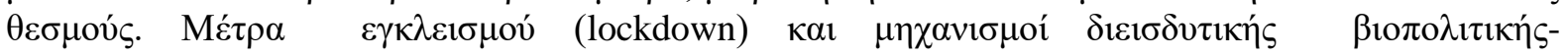

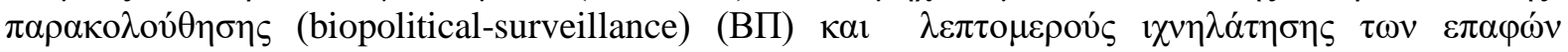

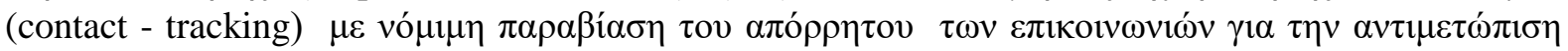

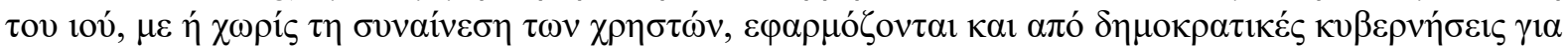

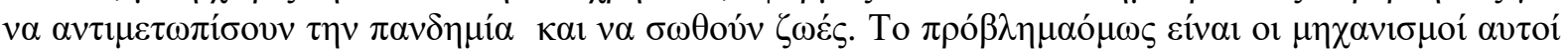

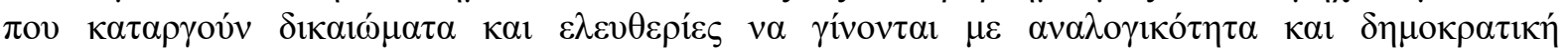

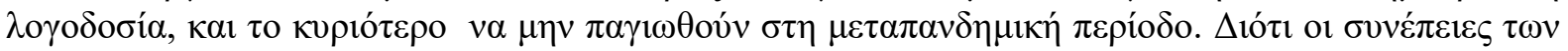

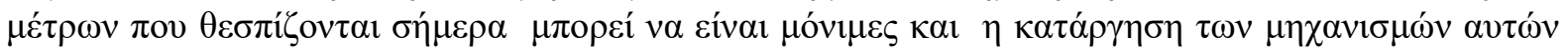

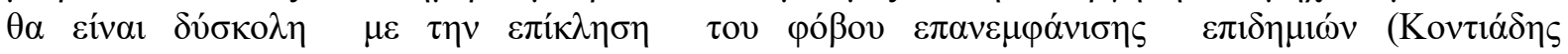

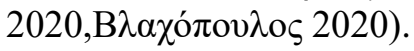

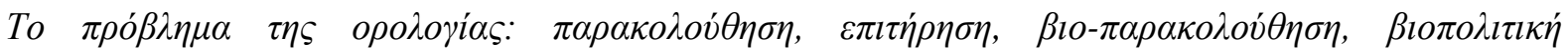
$\pi \alpha \rho \alpha \kappa о \lambda o v \theta \eta \eta \sigma \eta$ ( The problem of terminology)

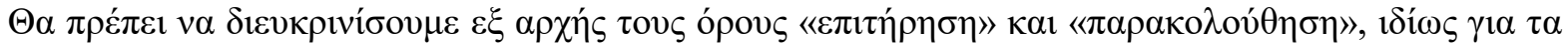

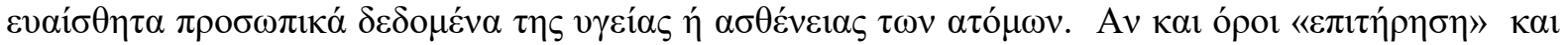

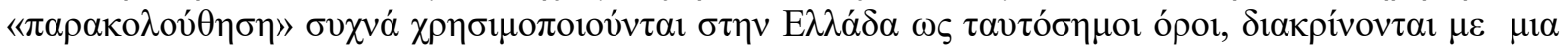

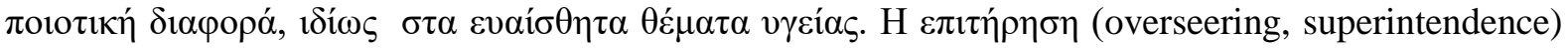

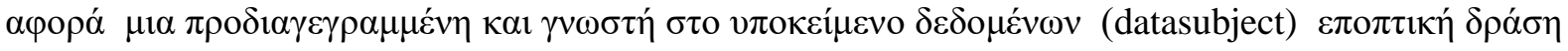




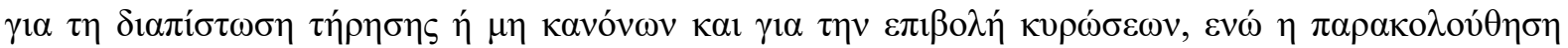

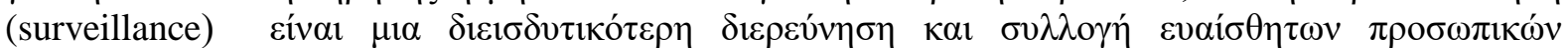

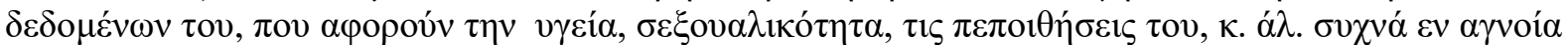

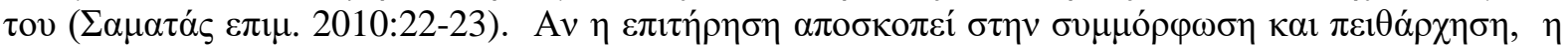

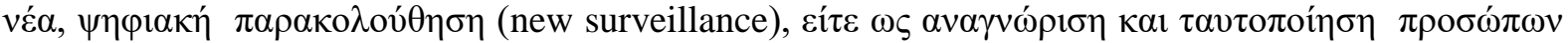

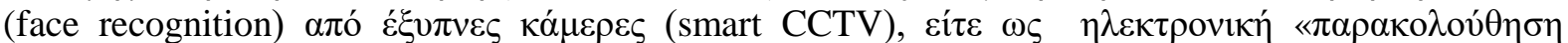

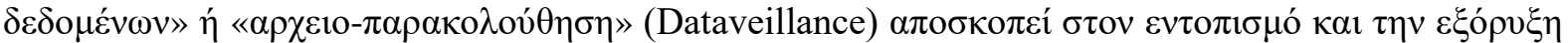

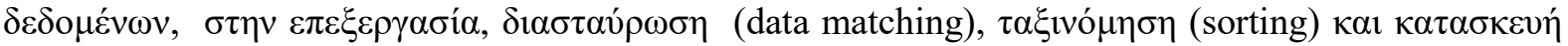

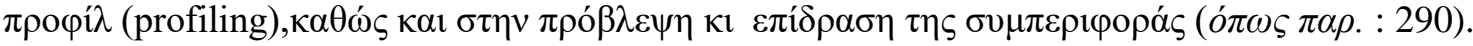

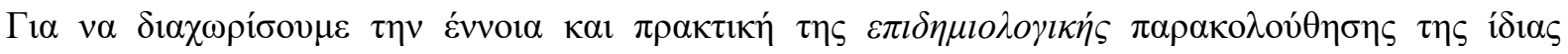

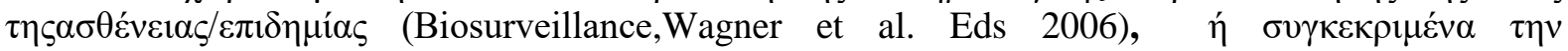

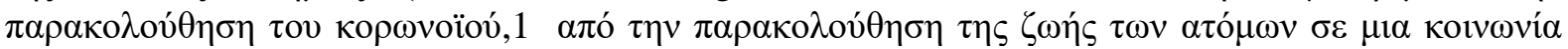
$\gamma 1 \alpha \quad \tau \eta \nu$ a

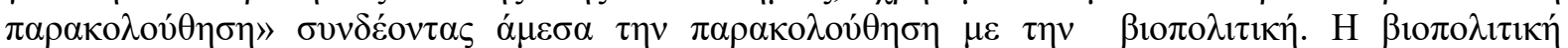

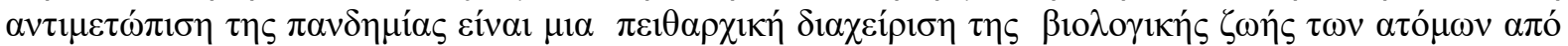

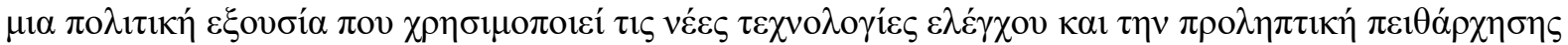

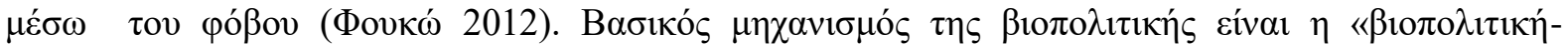

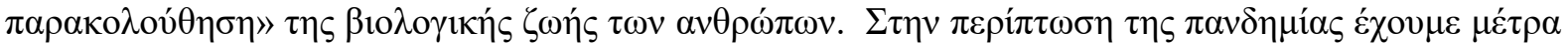

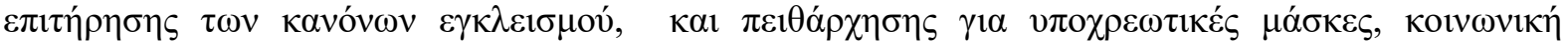

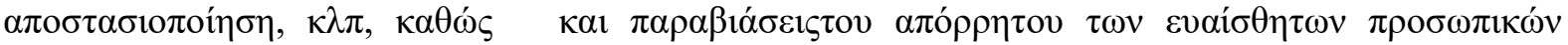

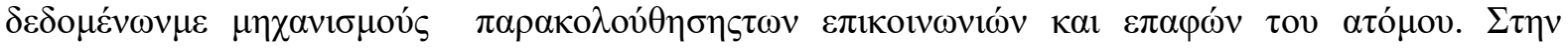

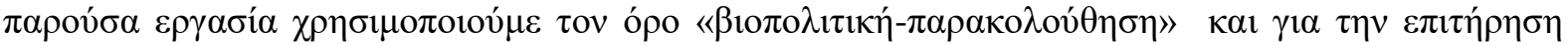

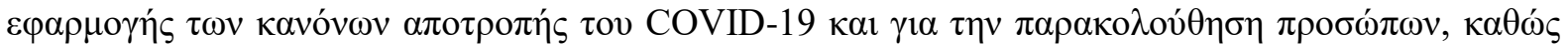

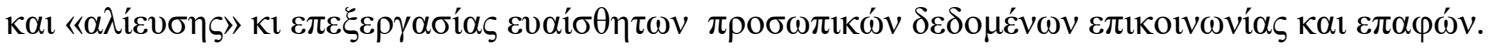

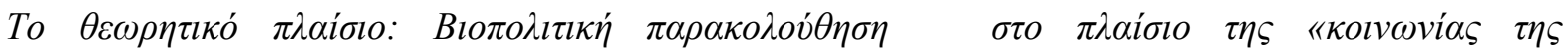

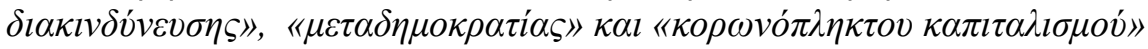

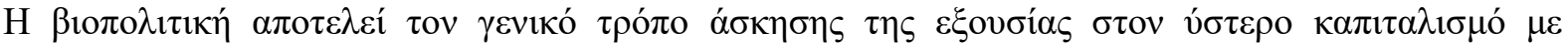

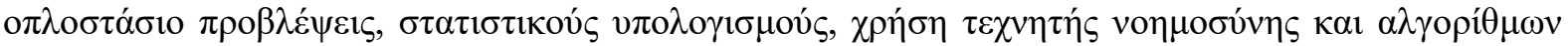

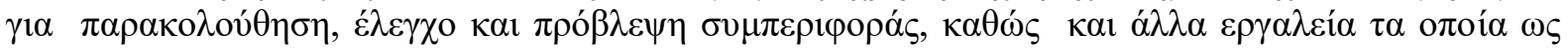

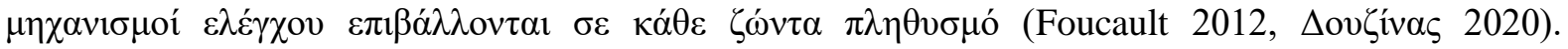

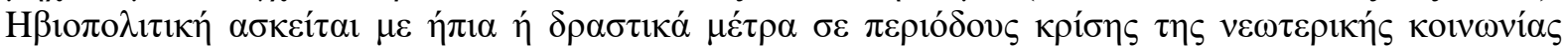

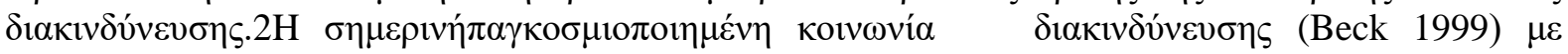

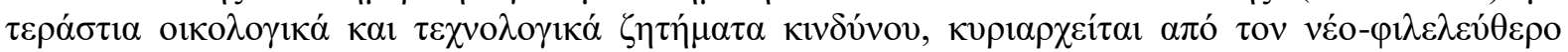

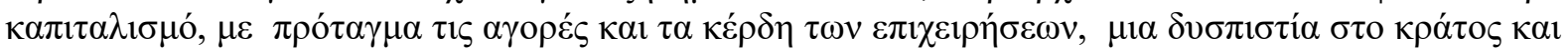

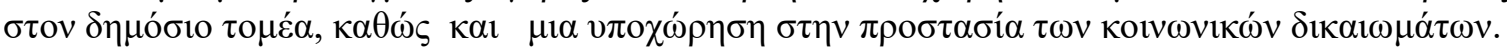

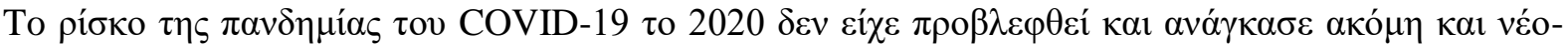


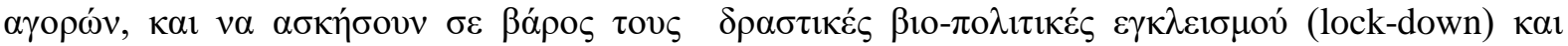

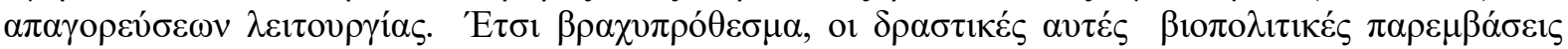

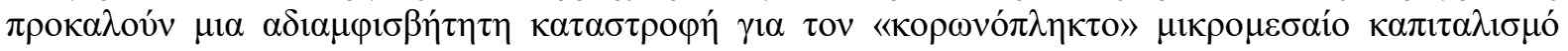
(Coronashock Capitalism, Ecks 2020).

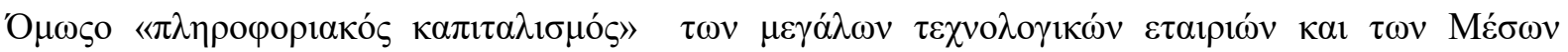

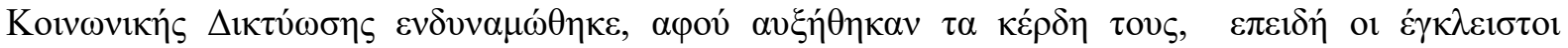

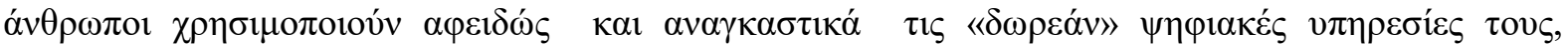

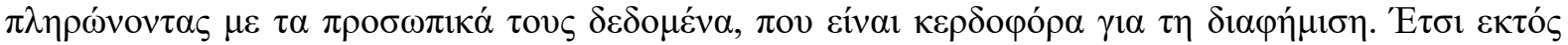

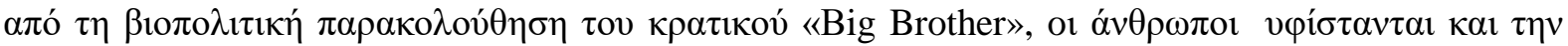

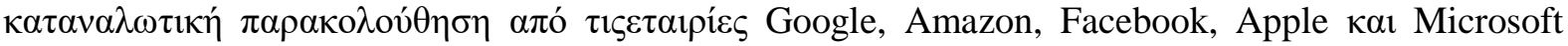

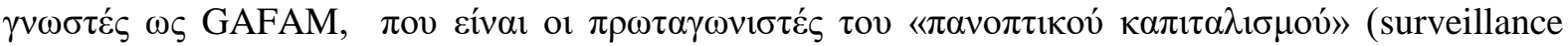

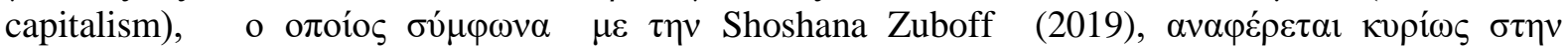

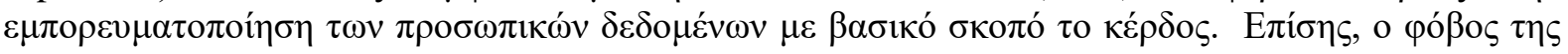

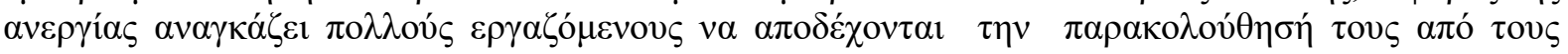

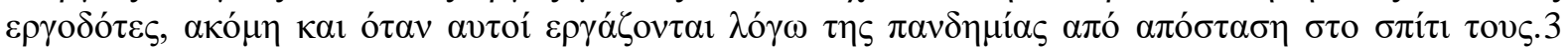

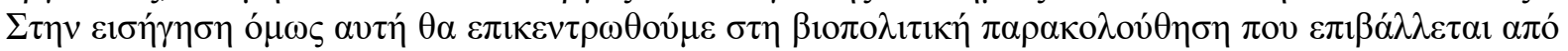

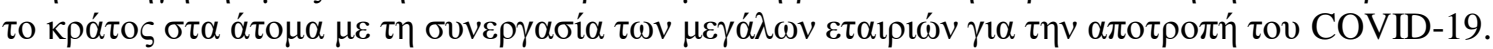




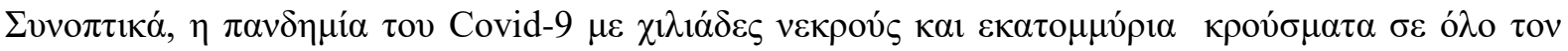

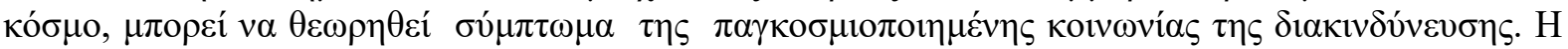

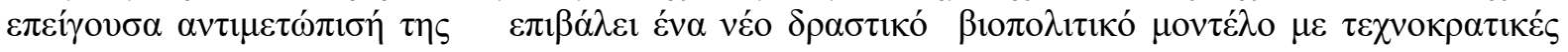

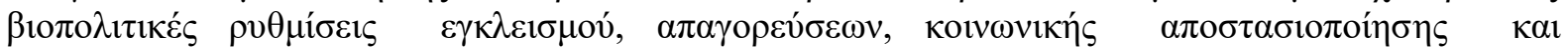

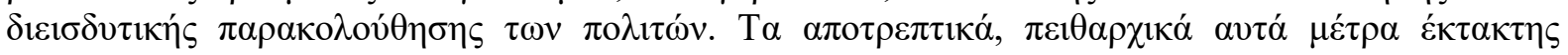

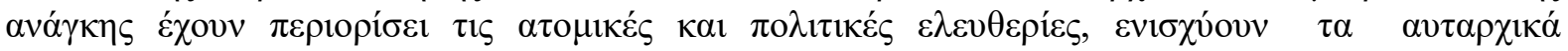

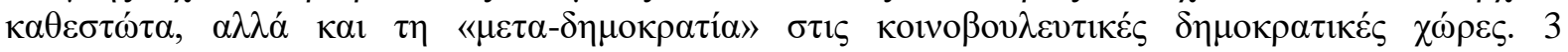

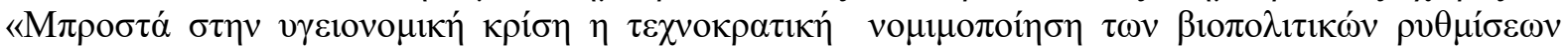

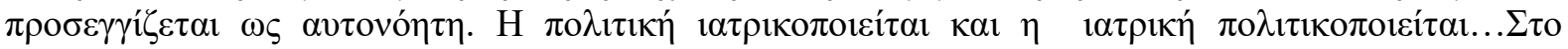

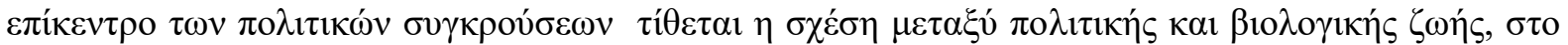

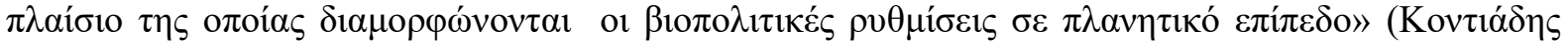
2020:49-50).

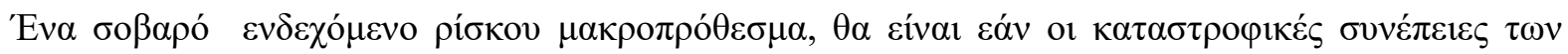

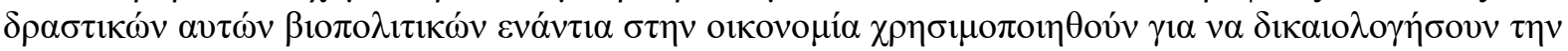

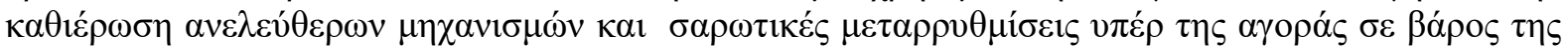

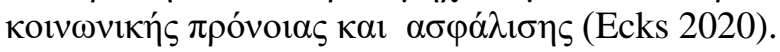

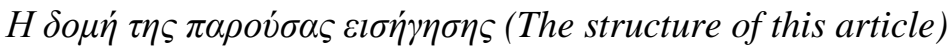

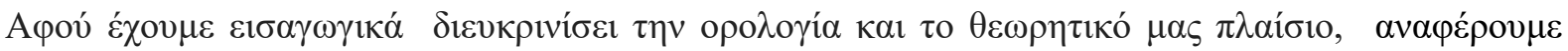

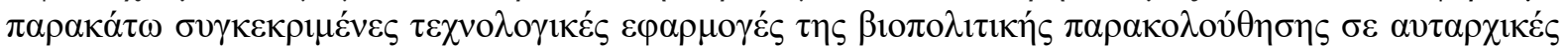

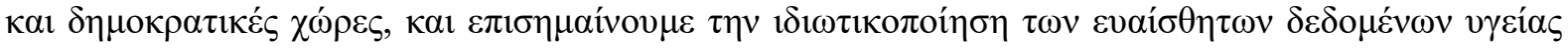

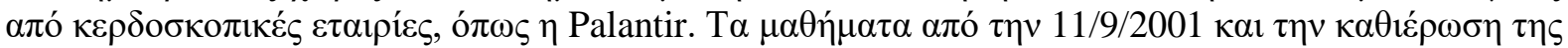

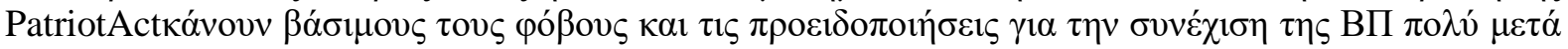

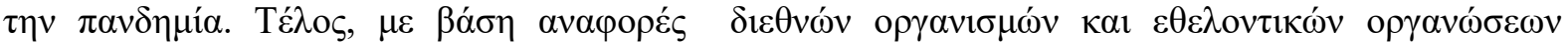

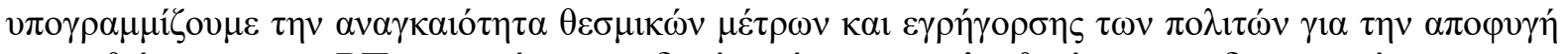

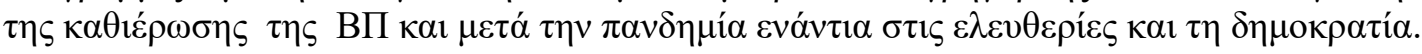

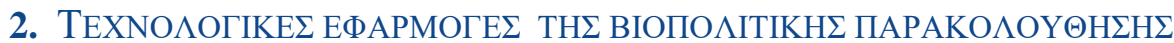

\section{(Technological apps of biopolitical surveillance)}

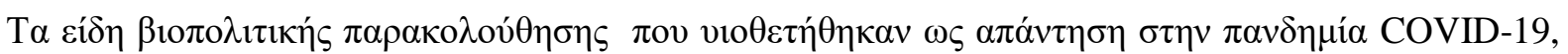

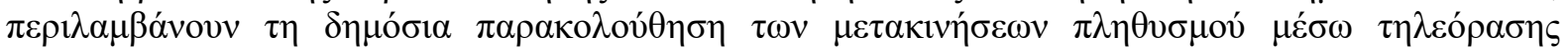

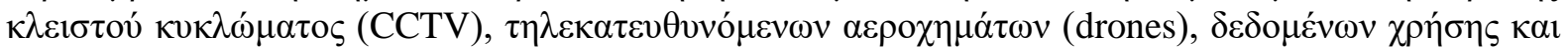

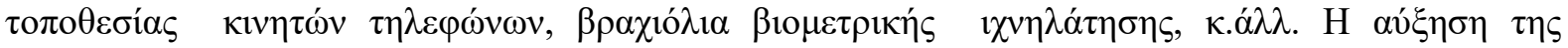

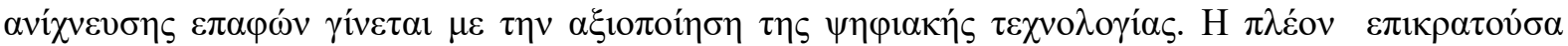

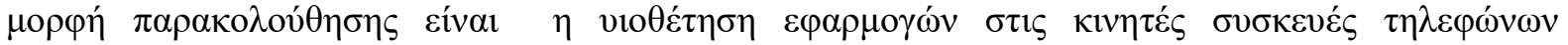

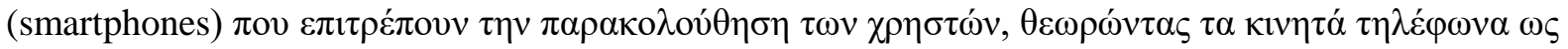

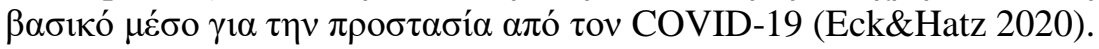

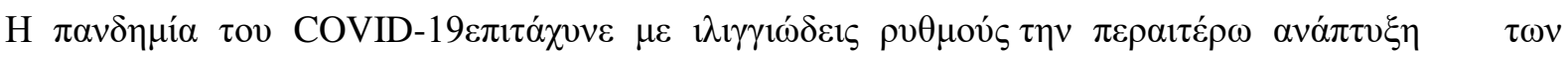

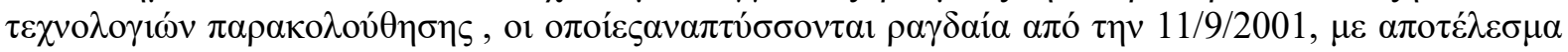

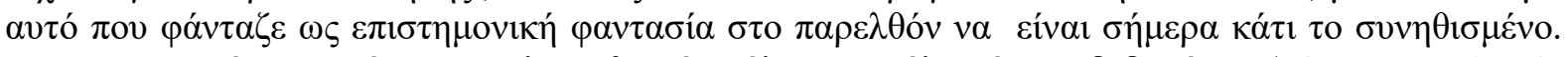

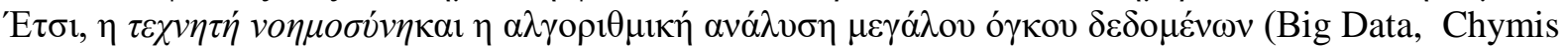

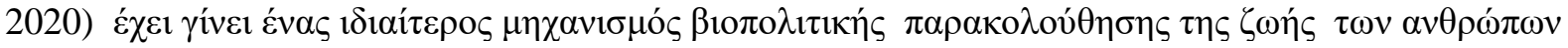

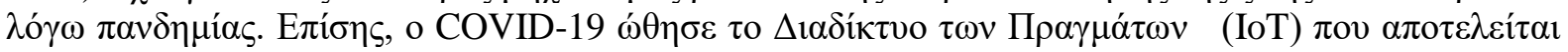

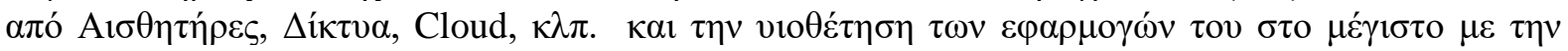

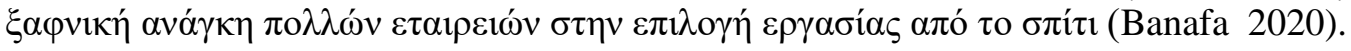

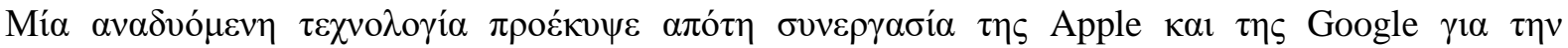

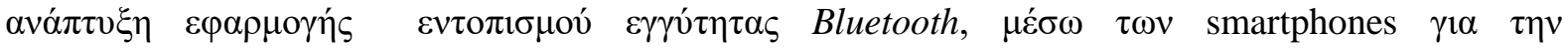

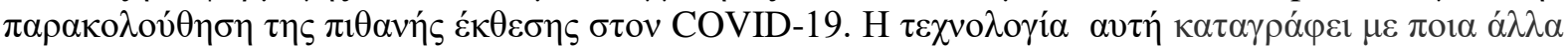

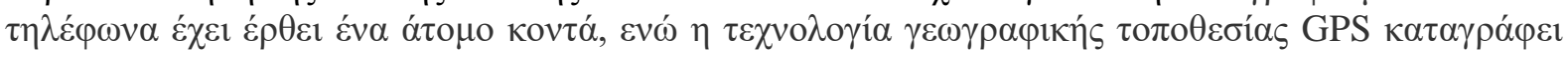

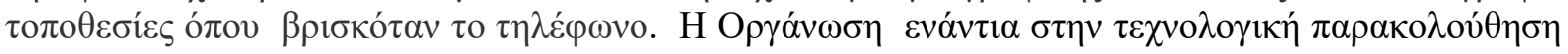

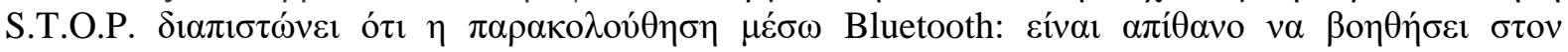

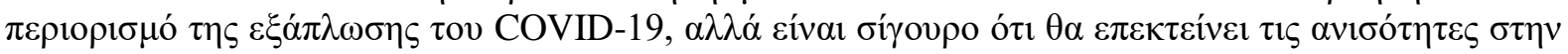

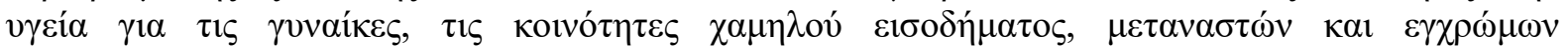
(https://www.stopspying.org/bluetooth ). 


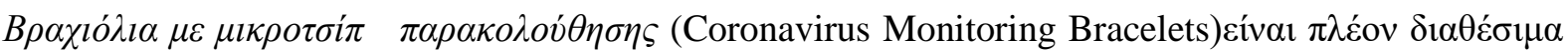

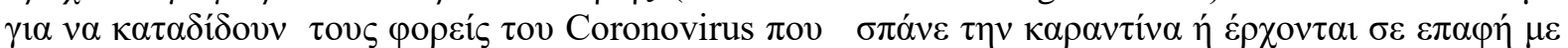

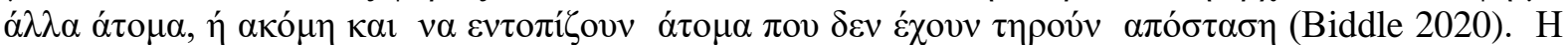

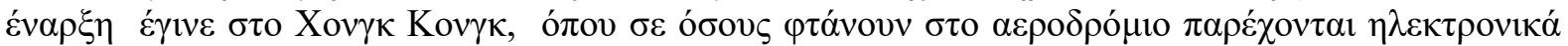

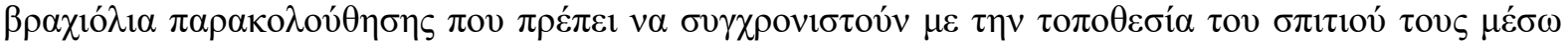

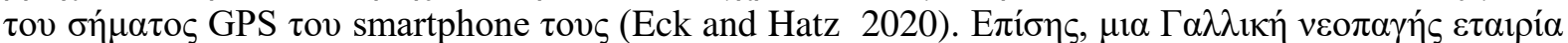

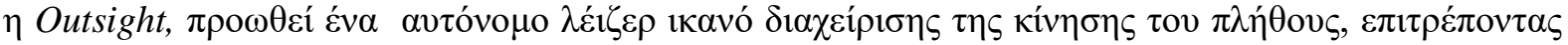

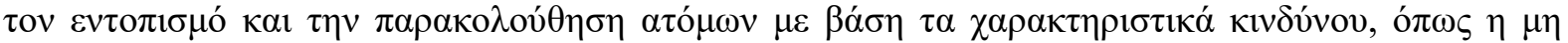

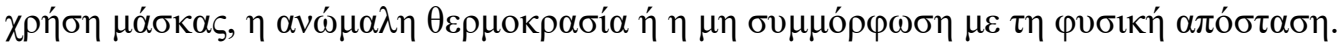

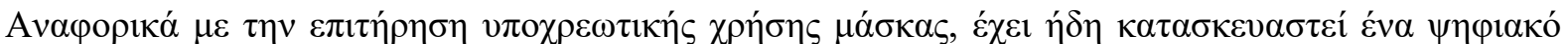

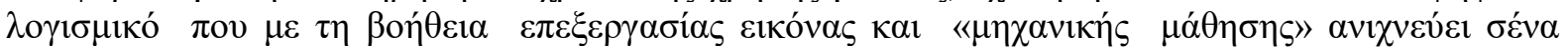

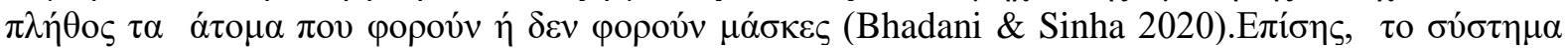

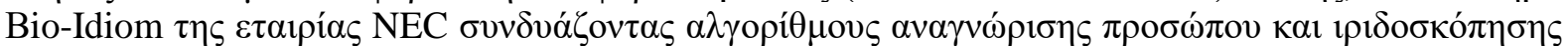

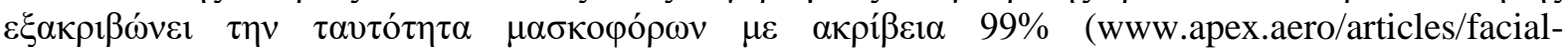
recognition-tech-works-masks).

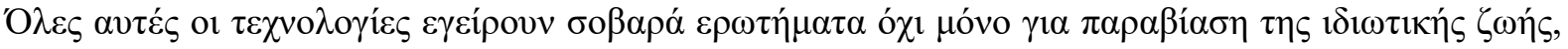

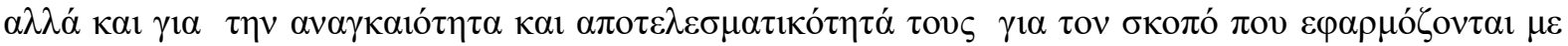

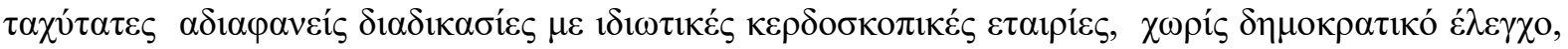

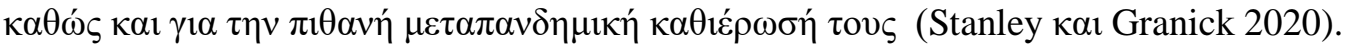

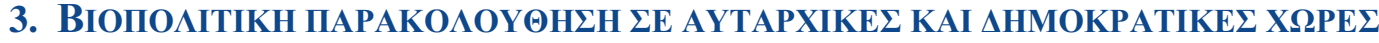

\section{(Biopolitical surveillance in authoritarian and democratic nations)}

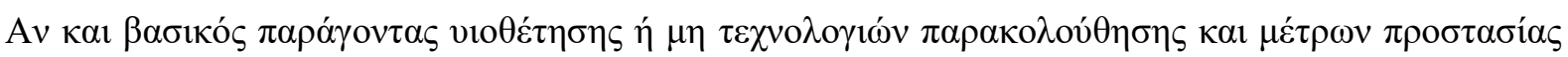

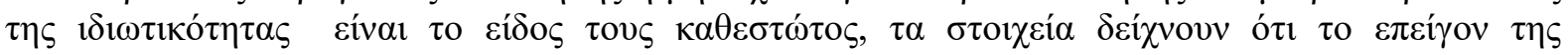

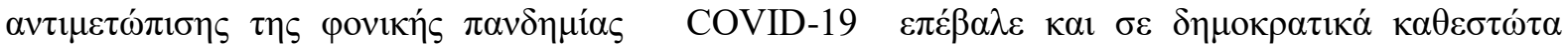

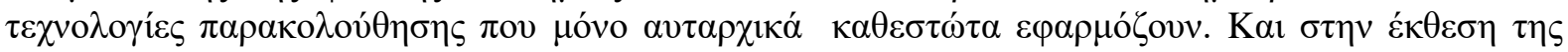

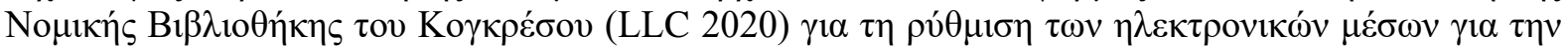

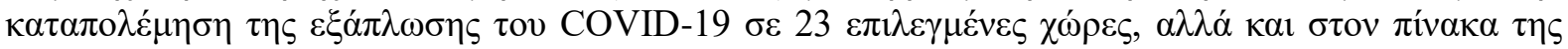

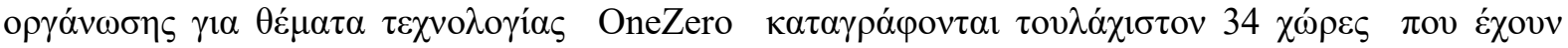

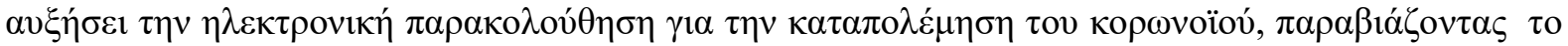

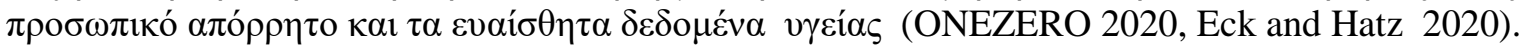

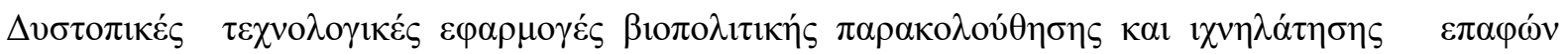

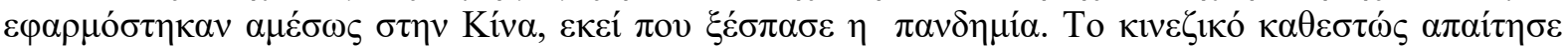

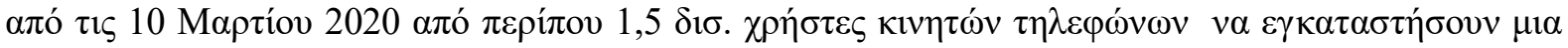

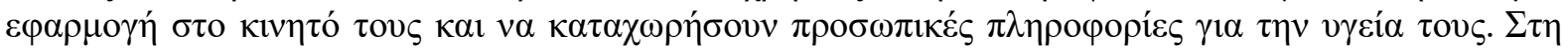

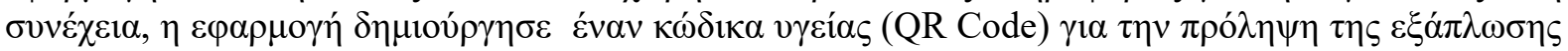

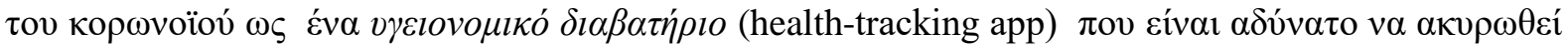

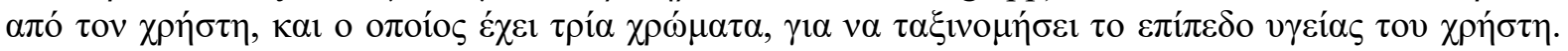

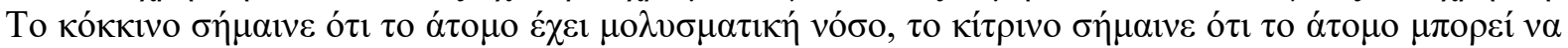

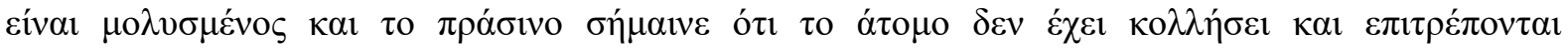

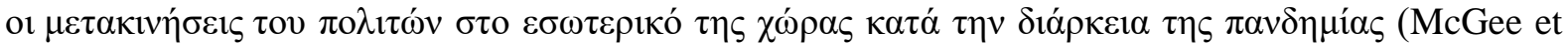
al.2020).

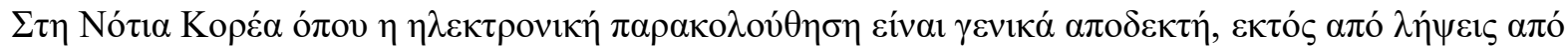

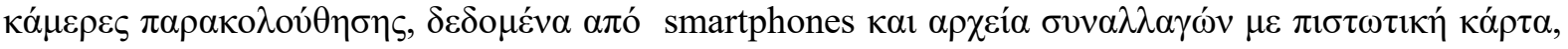

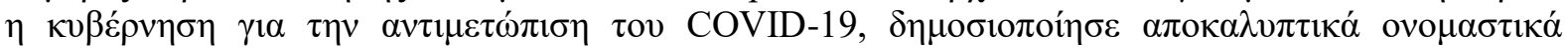

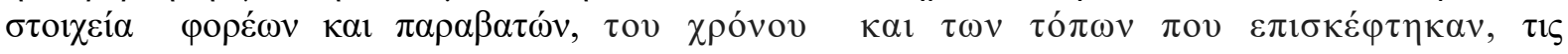

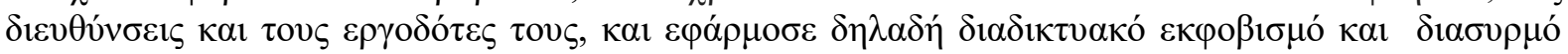
(LLC 2020).

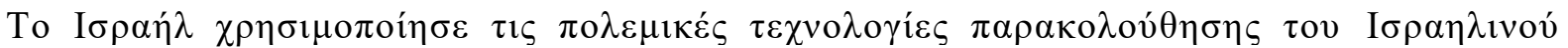

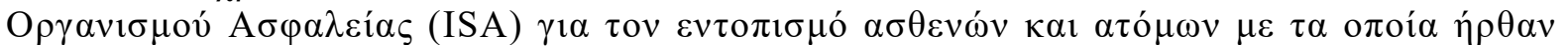

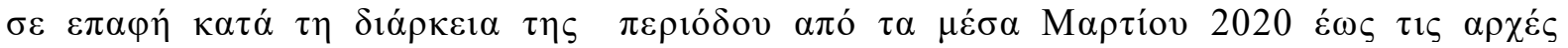

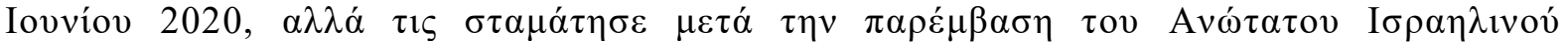

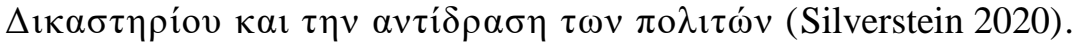




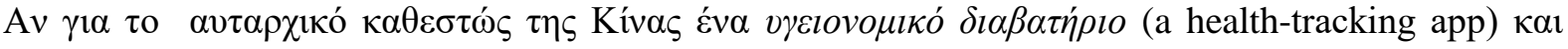

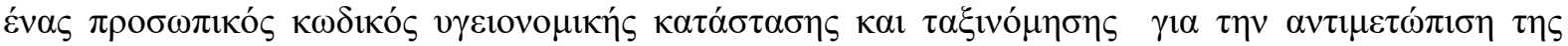

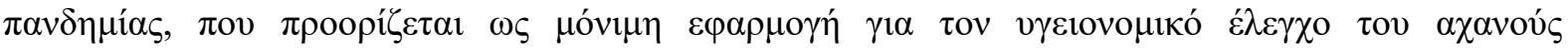

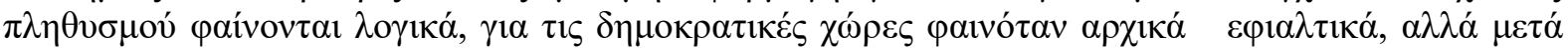

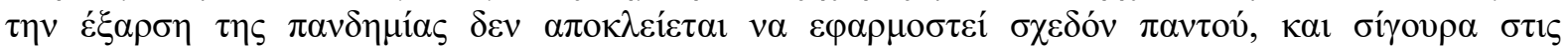

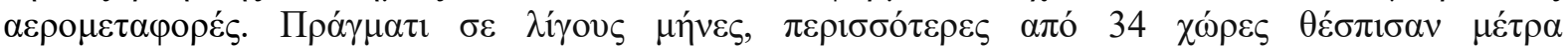

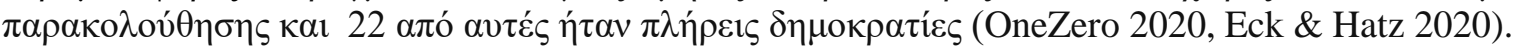

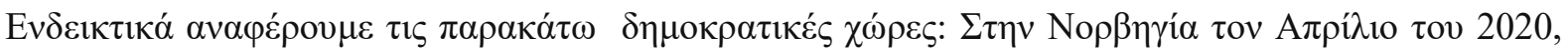

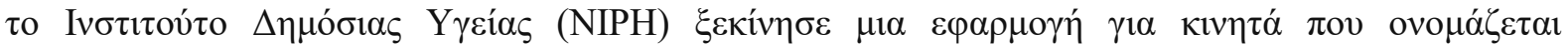

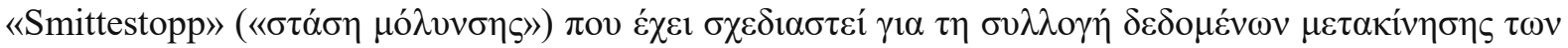

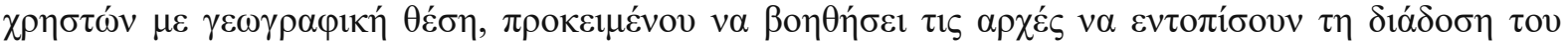

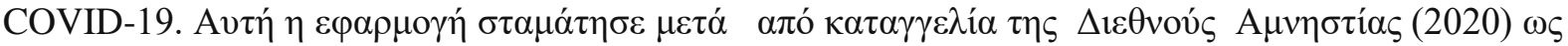

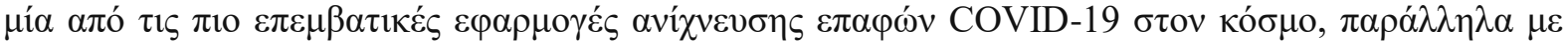

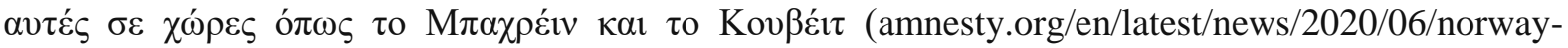
covid19-contact-tracing-app-privacy-win/ ).

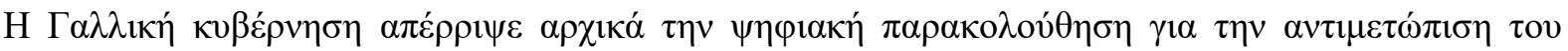

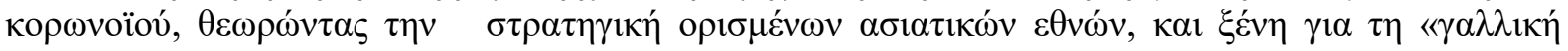

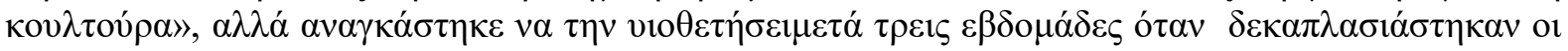

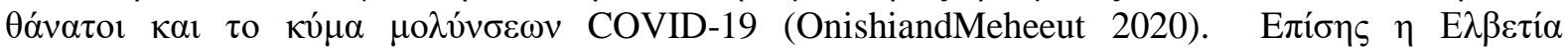

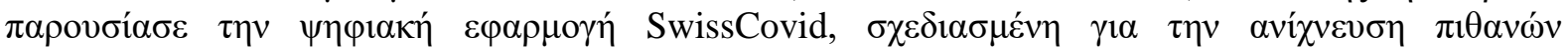

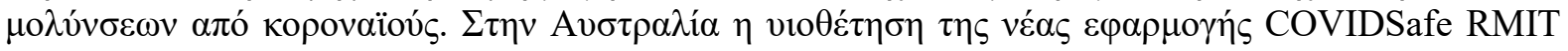

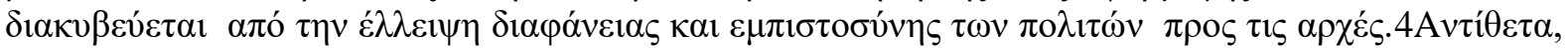

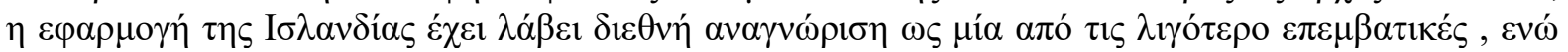

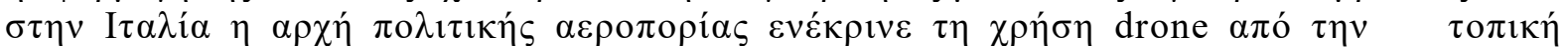

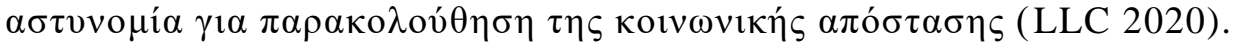

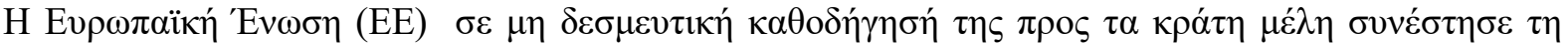

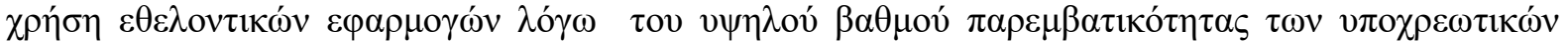

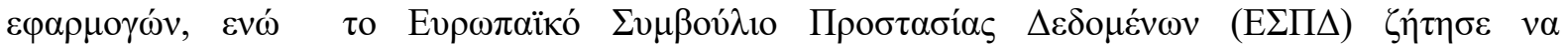

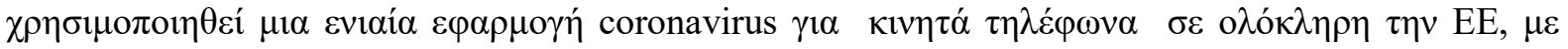

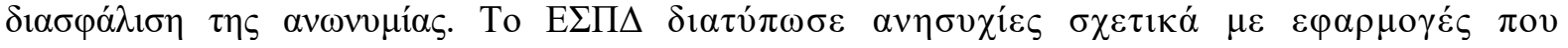

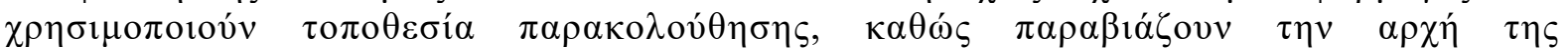

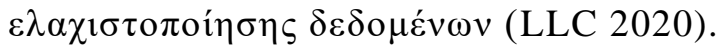

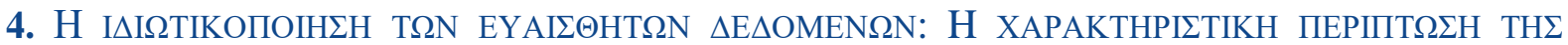 ETAIPIA PALANTIR}

(The privatization of sensitive (bio)-Data: The distinctive case of 'Palantir' corp.)

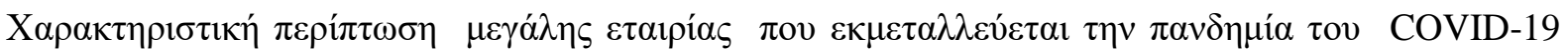

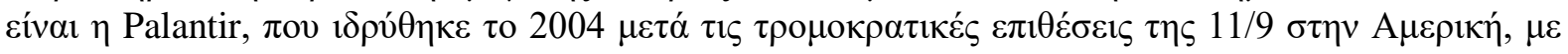

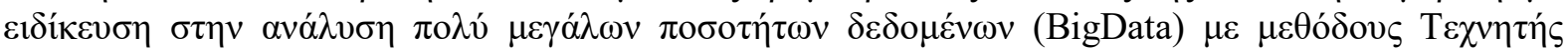

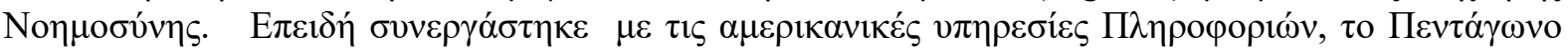

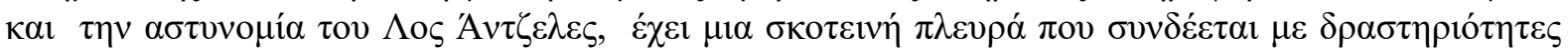

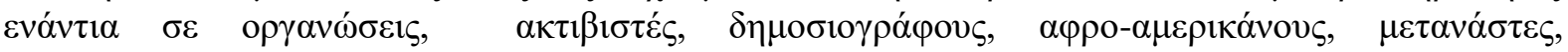

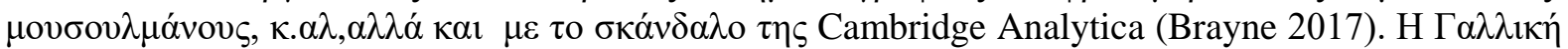

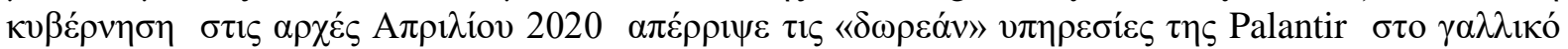

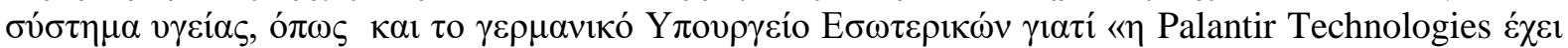

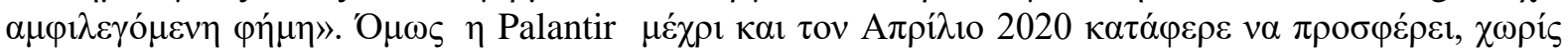

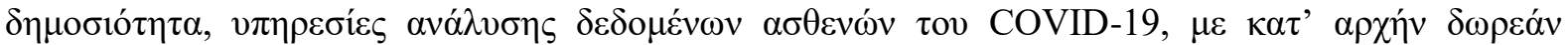

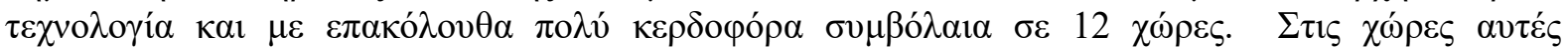

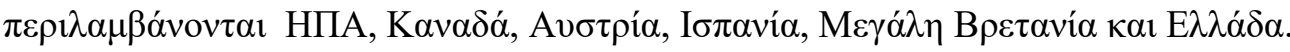

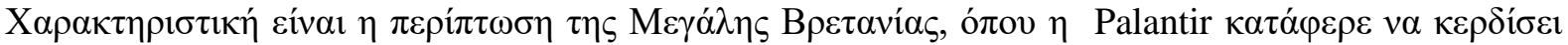

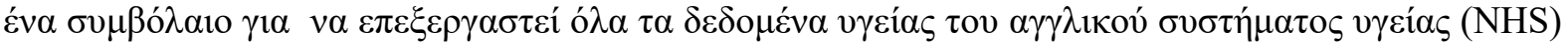

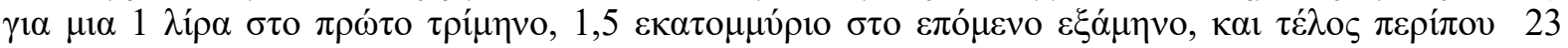

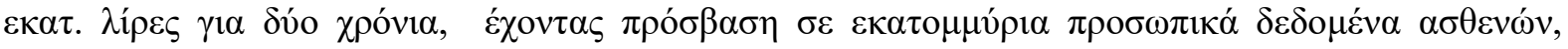




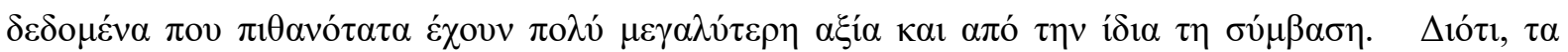

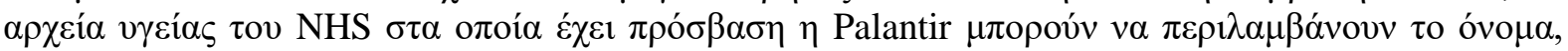

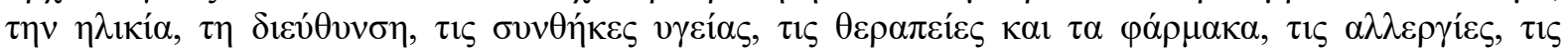

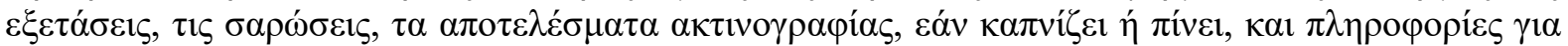

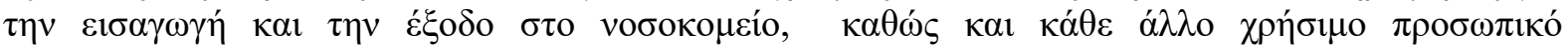

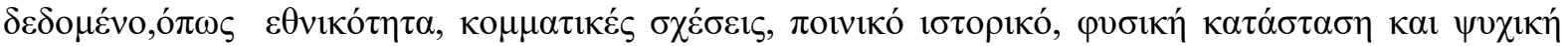

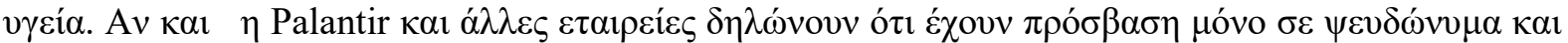

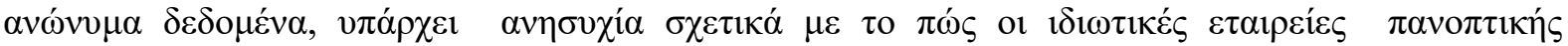

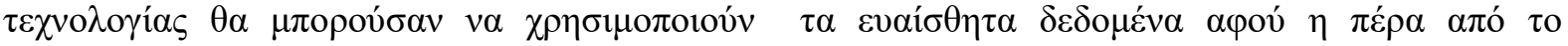

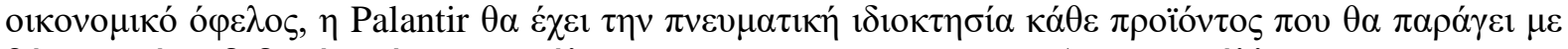

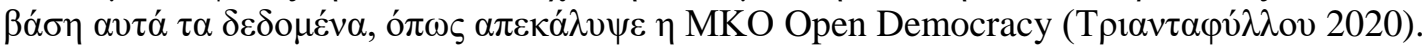

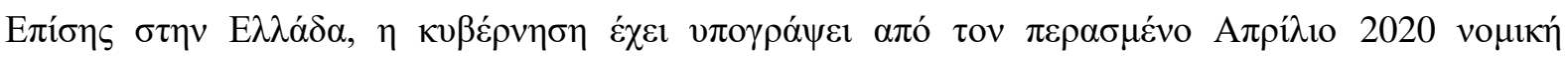

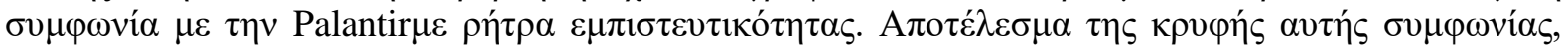

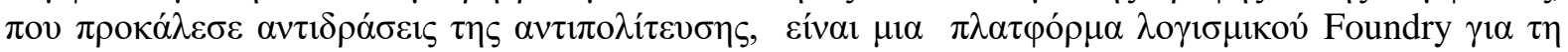

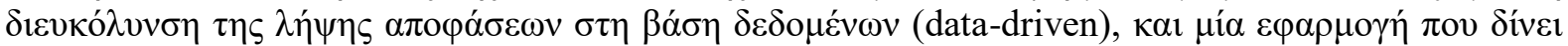

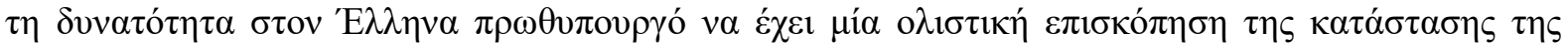

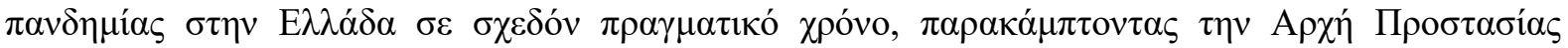

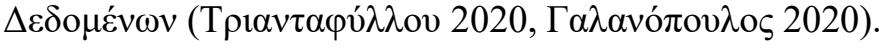

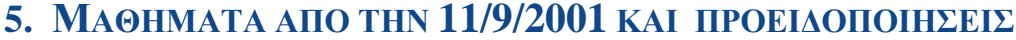

\section{( Lessons from 9/11/2001 and some warnings)}

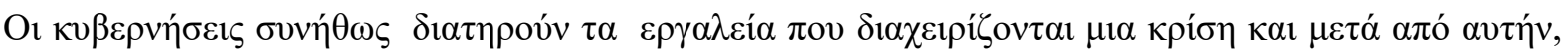

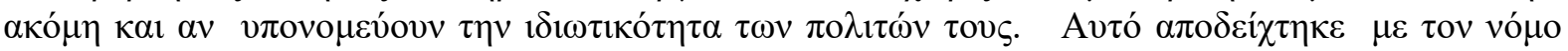

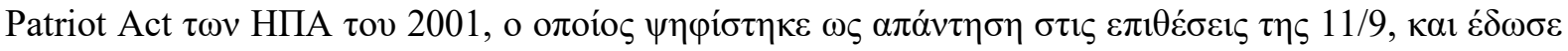

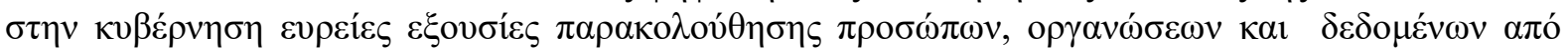

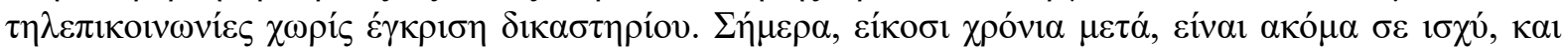

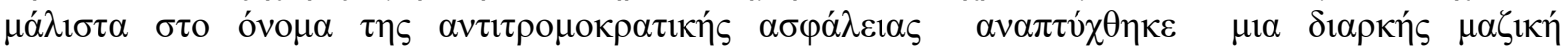

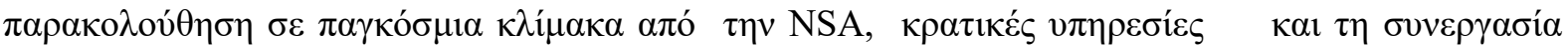

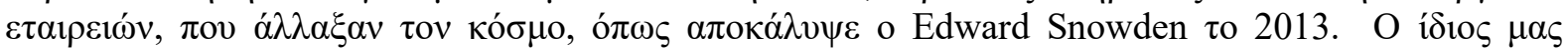

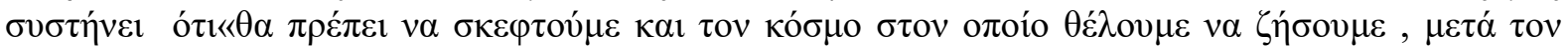

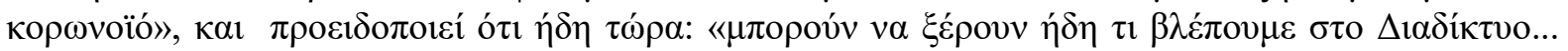

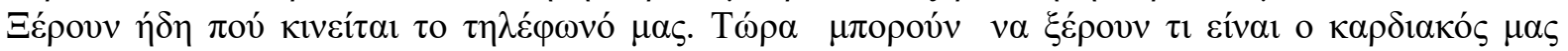

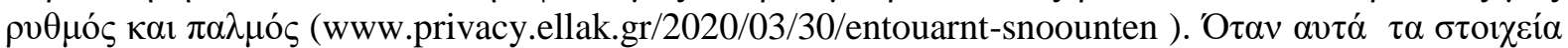

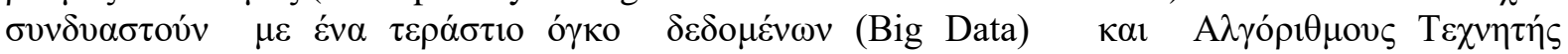

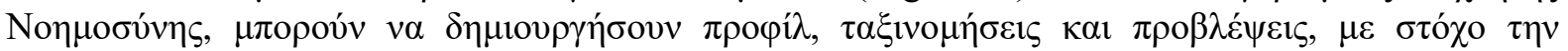

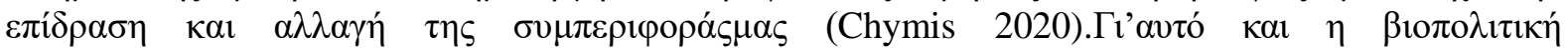

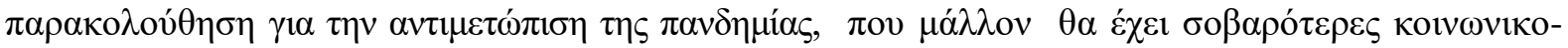

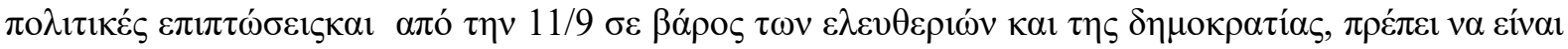

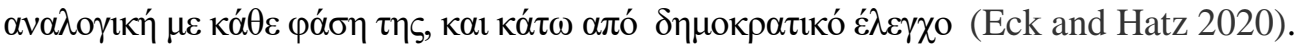

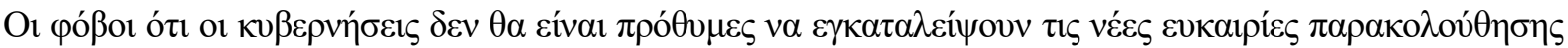

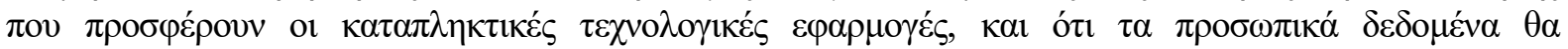

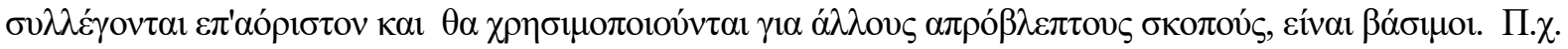

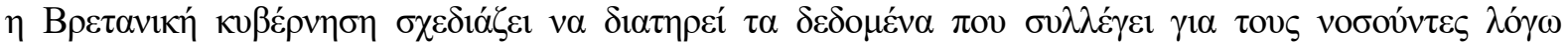

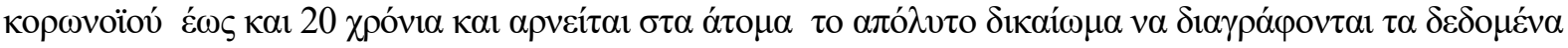

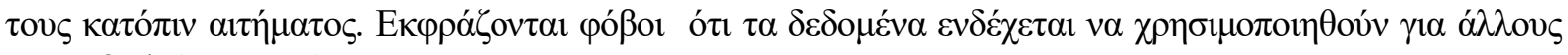
бколои́ (The Guardian, 28 May 2020).

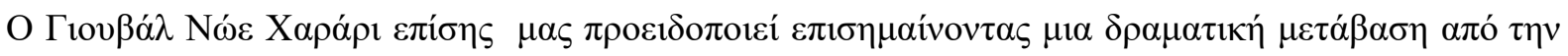

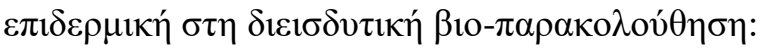

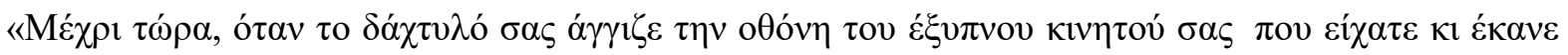

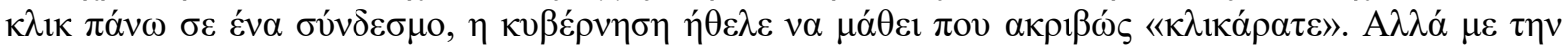

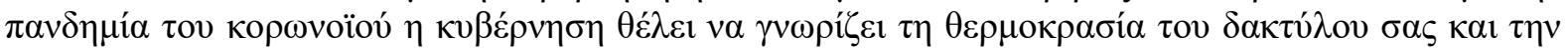

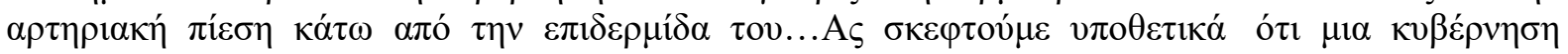

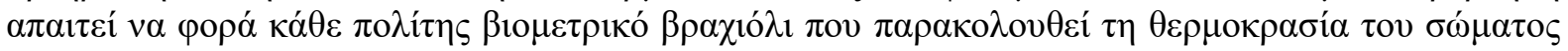




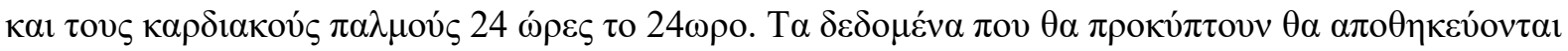

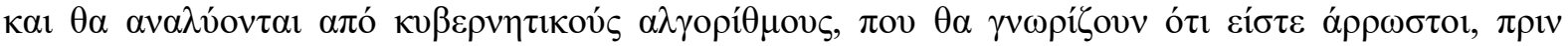

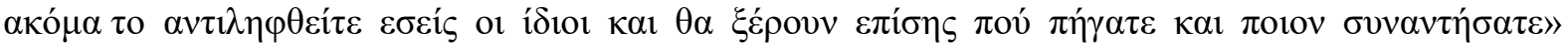
(Harari2020).

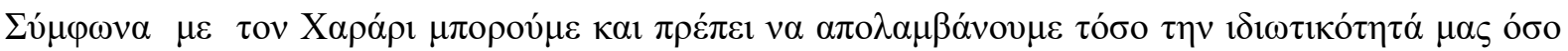

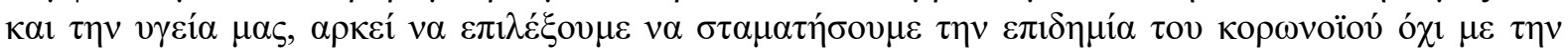

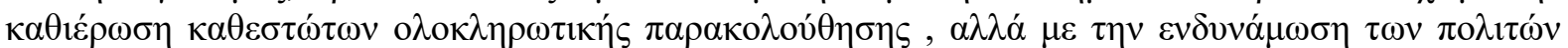

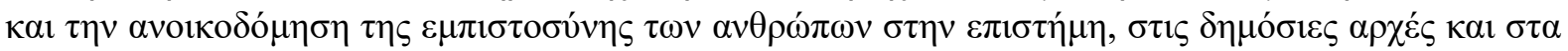
$\operatorname{MME}(\dot{o} \pi . \pi \alpha \rho$.).

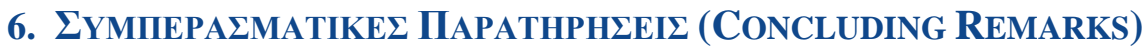

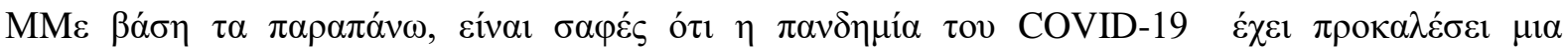

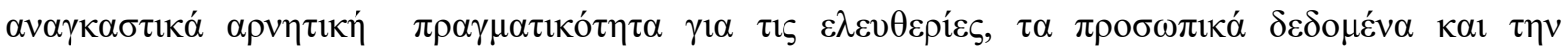

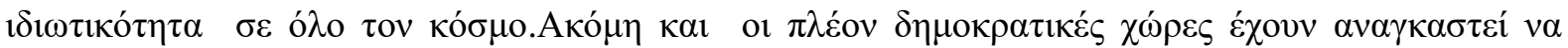

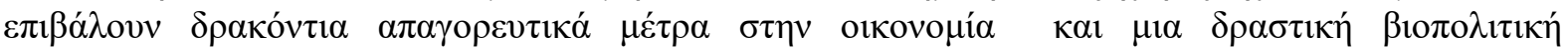

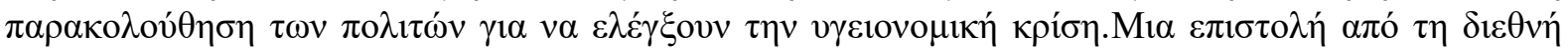

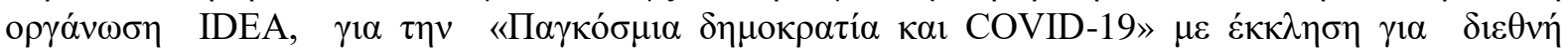

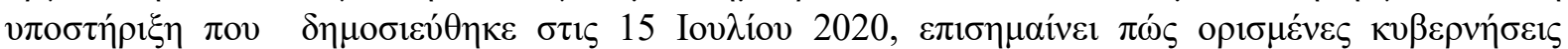

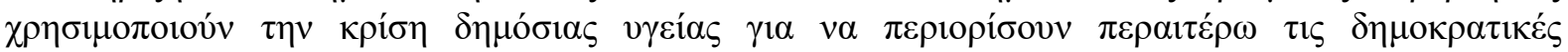

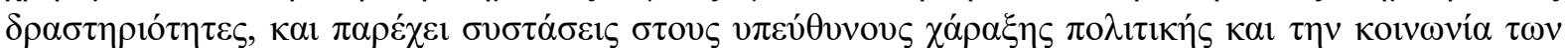

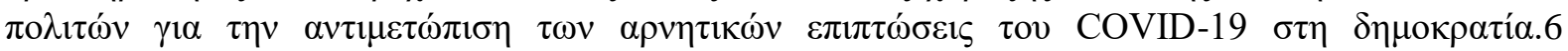

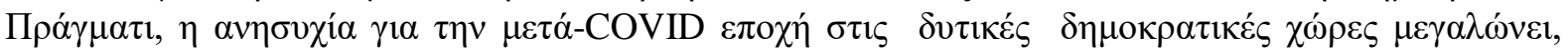

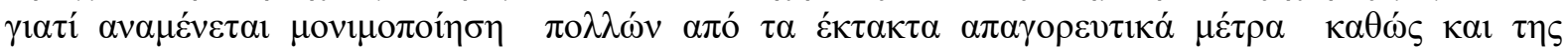

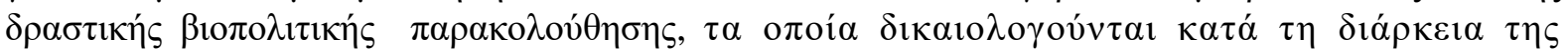

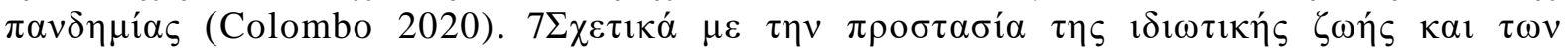

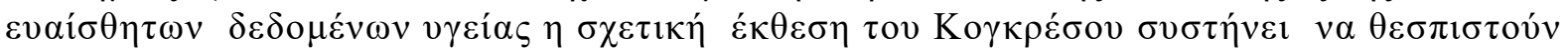

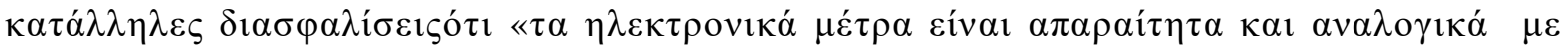

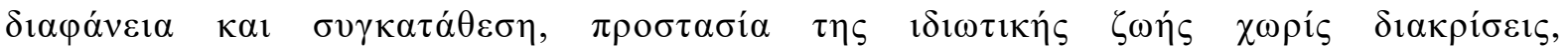

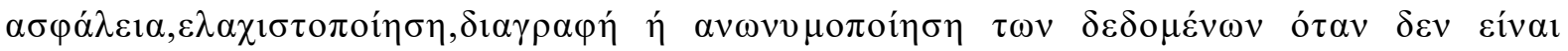

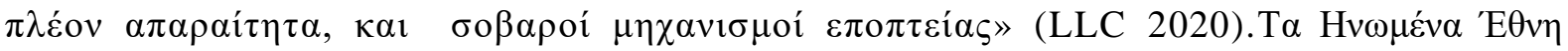

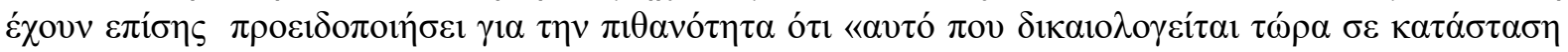

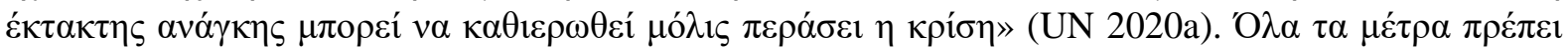

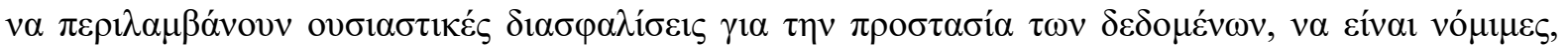

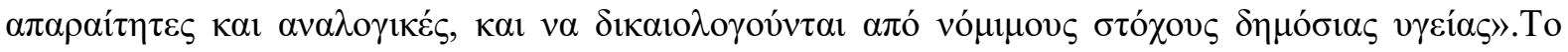

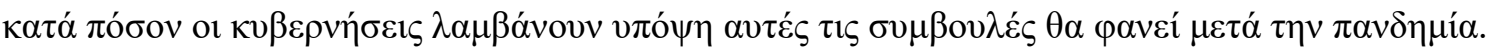

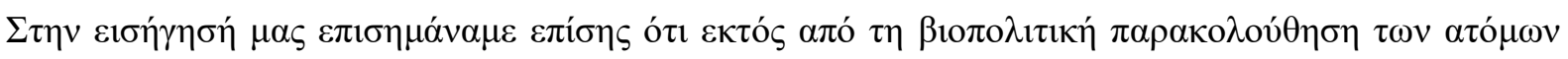

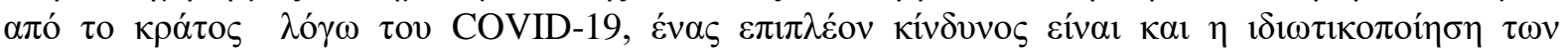

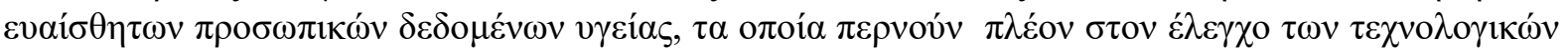

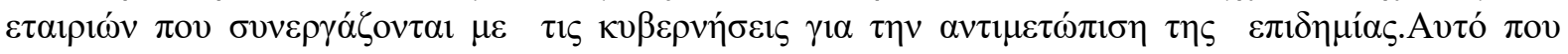

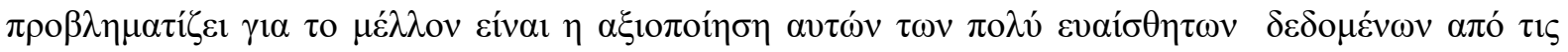

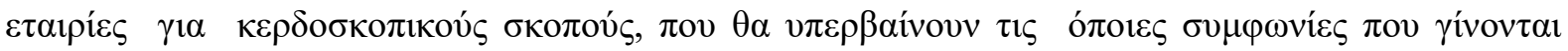

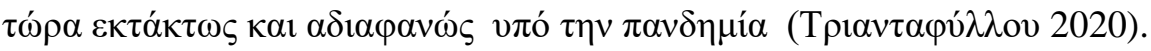

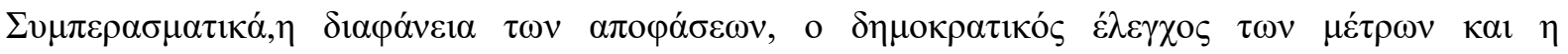

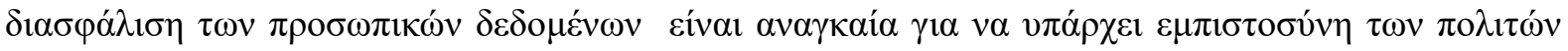

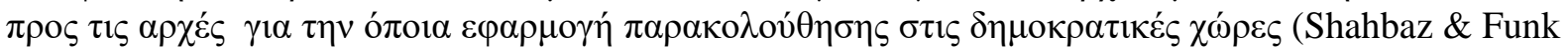

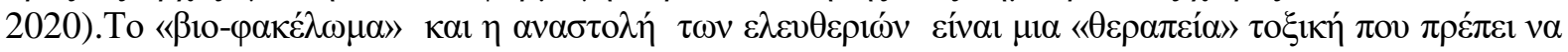

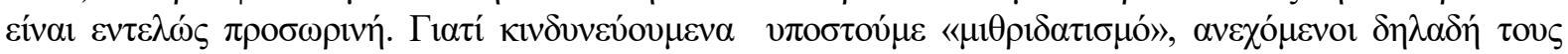

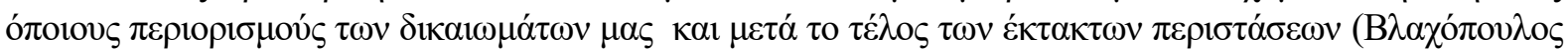

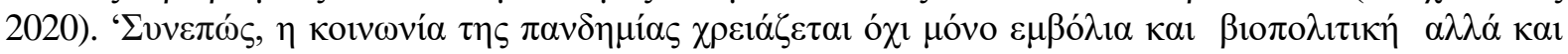

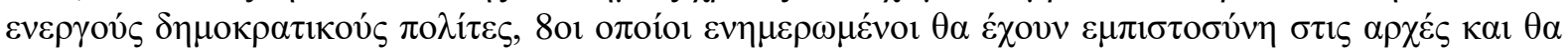

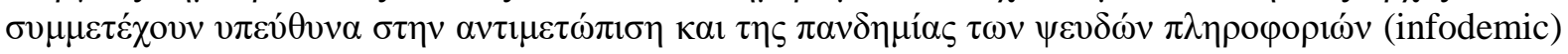


Biopolitical Surveillance Vs COVID-19 in Authoritarian and Democratic States and Its Possible PostPandemic Implications

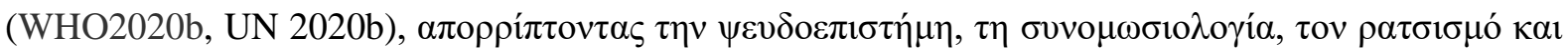
$\tau \eta v \xi \varepsilon v o \varphi \circ \beta i ́ \alpha$.

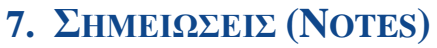

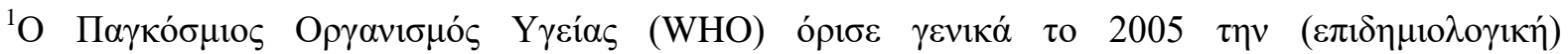

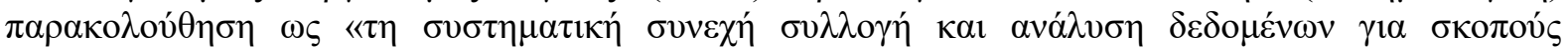

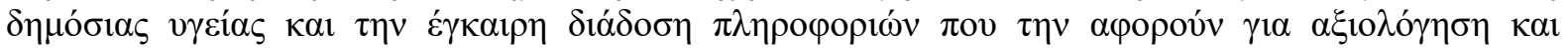

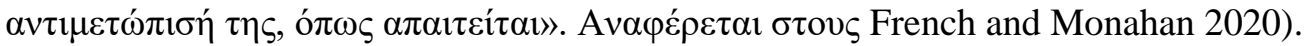

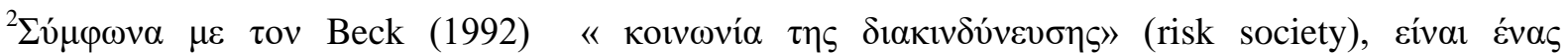

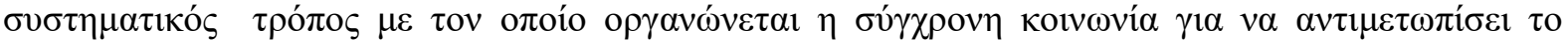

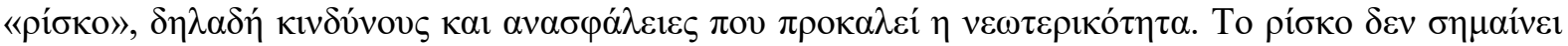

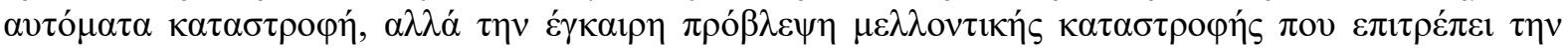

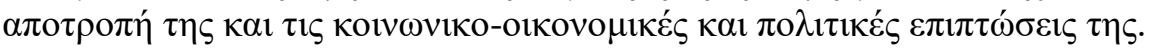

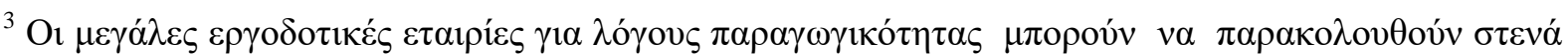

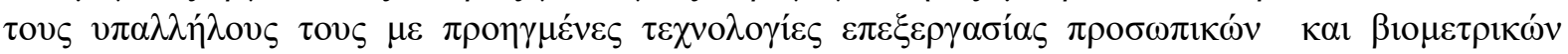

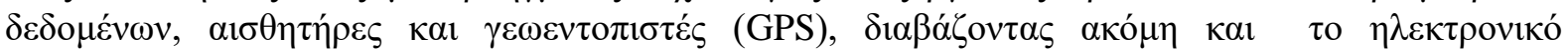
$\tau \alpha \chi 0 \delta \rho о \mu \varepsilon i ́ o ~ \tau o v \varsigma, ~ \kappa \lambda \pi$ (Dreyfuss 2020).

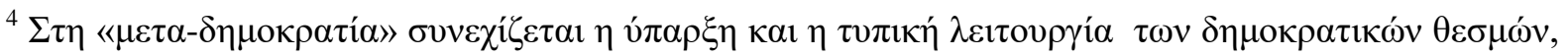

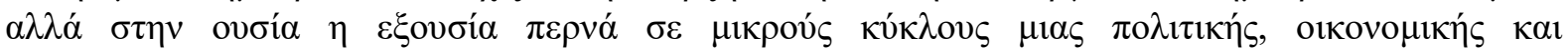

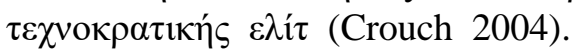

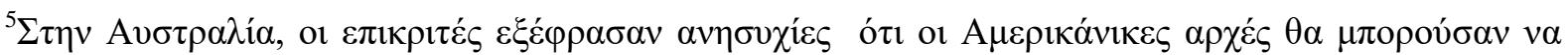

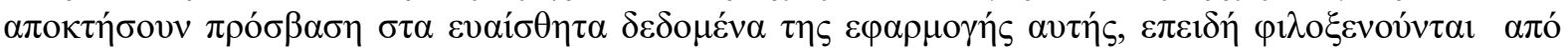

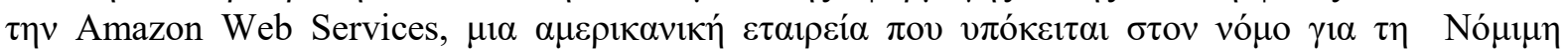

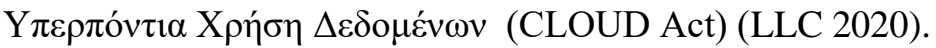

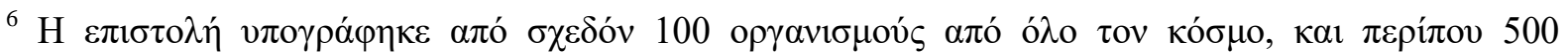

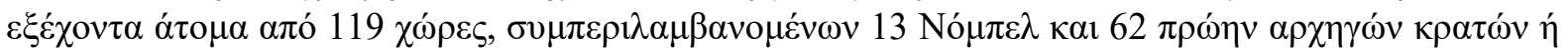
$\kappa v \beta \varepsilon \rho v \eta ́ \sigma \varepsilon \omega v$, (https://www.idea.int/news-media/multimedia-reports/call-defend-democracy).

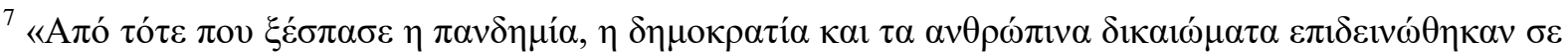

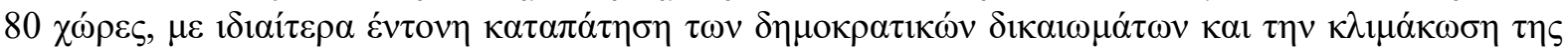

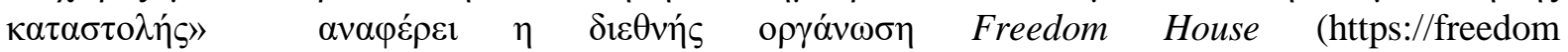
house.org/issues/democracy-during-pandemic).

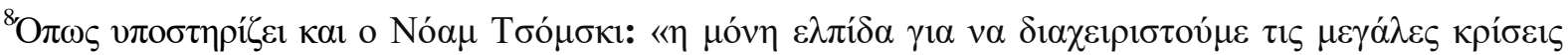

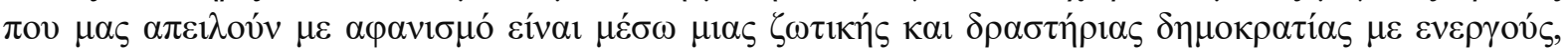

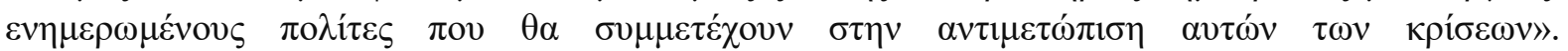

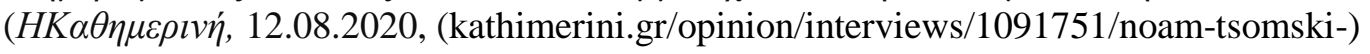

\section{ANATOPE (REFERENCES)}

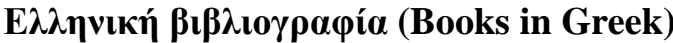

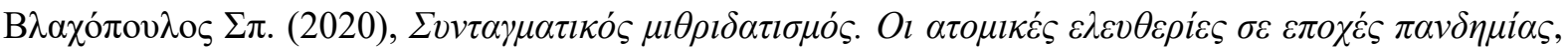

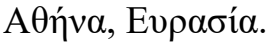

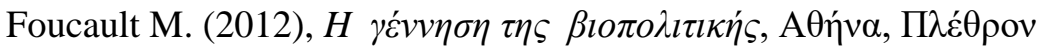

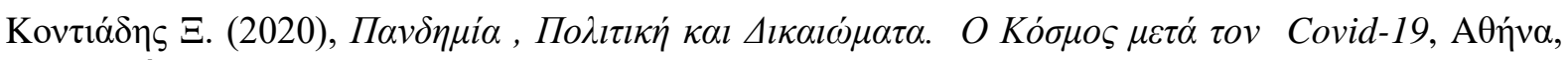

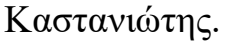

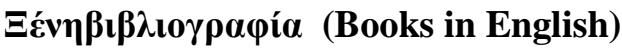

Crouch C. (2004), Post- Democracy, Cambridge, Polity Press

Wagner M. et al. Eds (2006), Handbook of Biosurveillance, London, Elsevier

Zuboff S. (2019), The Age of Surveillance Capitalism: The Fight for a Human Future at the New Frontier of Power, N.Y., Public Affairs. 
Biopolitical Surveillance Vs COVID-19 in Authoritarian and Democratic States and Its Possible PostPandemic Implications

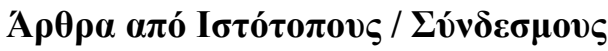

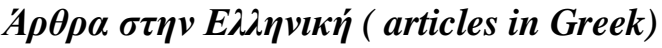

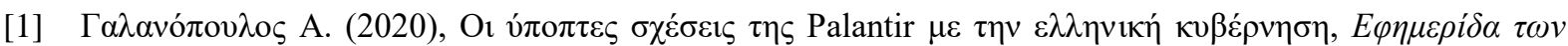

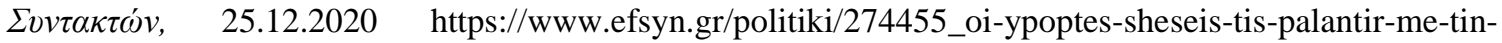
elliniki-kybernisi

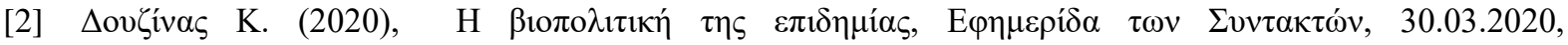
https://tinyurl.com/y4bp97dj

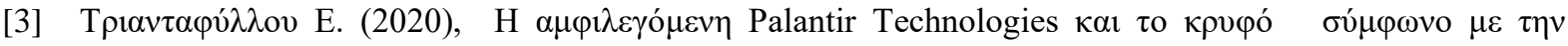

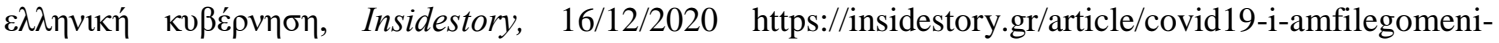
palantir-synergasia-elliniki- kyvernisi

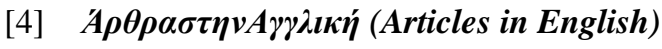

[5] Bhadani A.K. and Sinha A. (2020), A facemask detector using machine learning and image processing techniques. https://www.researchgate.net/publication/345972030

[6] Biddle S.(2020), Coronavirus Monitoring Bracelets Flood the Market, Ready to Snitch on People Who Don't Distance. https://theintercept.com/2020/05/25/coronavirus-tracking-bracelets-monitors-surveillancesupercom/

[7] Brayne S. (2017), Big Data Surveillance: The Case of Policing

[8] American Sociological Review 2017, Vol. 82(5) 977-1008 https://doi.org/10.1177/0003122417725865

[9] Chymis, A. (2020), Artificial Intelligence in the post-COVID-19 era. Homo Virtualis, 3(2), 55-67. doi: https://doi.org/10.12681/homvir.25449

[10] Colombo E. (2020), Human Rights-inspired Governmentality. COVID-19 through a Human Dignity Perspective, Critical Sociology, 1-11,https://doi.org/10.1177/0896920520971 846

[11] Dreyfuss J. (2020), Here's how employers are using tech tools to keep a close watch on their remote workers. CNBC June 24, 2020 https://www.cnbc.com/2020/06/24/new-tech-tools-employers-are-using-tokeep watch-on-remote-workers.html

[12] Eck K. and Hatz S. (2020), State surveillance and the COVID-19 crisis, Journal of Human Rights, 19:5, 603-612, https://www.tandfonline.com/doi/full/10.1080/14754835.2020.1816163?scroll=top\&need Access $=$ true

[13] Ecks St. (2020), Coronashock Capitalism: The Unintended Consequences of Radical Biopolitics, Medical Anthropology Quarterly, Volume 34, Issue 3 (Sept 2020), http://medanthroquarterly.org/2020/04/06/ coronashock-capitalism-the-unintended-consequences-of-radical-biopolitics/

[14] French M. and Monahan T. (2020). Dis-ease Surveillance: How Might Surveillance Studies address COVID-19, Surveillance \& Society 18(1): 1-11.

[15] https://ojs.library.queensu.ca/index.php/surveillance-and-society/index

[16] Harari N.Y. (2020), The world after coronavirus. Financial Times, March 20, 2020, https://www.ft.com/content/19d90308-6858-11ea-a3c9-1fe6fedcca75

[17] Law Library of Congress (LLC 2020), Regulating Electronic Means to Fight the Spread of COVID-19, LL File No. 2020-019000,June 2020. https://www.loc.gov/law/help/coronavirus-apps/coronavirusapps.pdf

[18] McGee, H. Murphy, P., and Bradshaw, T. (2020), Coronavirus apps: The risk of slipping into a surveillance state, Financial Times, 27 April 2020. https://www.ft.com/content/d2609e26-8875-11ea-a01ca28a3e3fbd33

[19] ONEZERO (2020), We Mapped How the Coronavirus is Driving New Surveillance Programs Around the World, https://onezero.medium.com/the-pandemic-is-a-trojan-horse-for-surveillance-programs-aroundthe-world-887fa6f12ec9

[20] Onishi N. and Meheeut C. (2020), France Weighs Its Love of Liberty in Fight Against Coronavirus, NYT, April 17, 2020. https://www.nytimes.com/2020/04/17/world/europe/coronavirus-france-digitaltracking.html?searchResultPosition $=5$

[21] Shahbaz A. and Funk A. (2020), False Panacea: Abusive Surveillance in the Name of Public Health. https://freedomhouse.org/report/report-sub-page/2020/false-panacea-abusive-surveillance-name-publichealth

[22] Silverstein, R. (2020), Israel is militarising and monetising the COVID-19 pandemic, The Wire, 20 April. https://thewire.in/world/israel-is-militarising-and-monetising-the-covid-19-pandemic

[23] Stanley J., Granick, Stiusa J. (2020), The Limits of Location Tracking in an Epidemic. https://www.aclu .org/report/aclu-white-paper-limits-location-tracking-epidemic (18 June 2020).

International Journal of Humanities Social Sciences and Education (IJHSSE)

Page $\mid 50$ 
Biopolitical Surveillance Vs COVID-19 in Authoritarian and Democratic States and Its Possible PostPandemic Implications

[24] UNITED NATIONS (UN 2020a), COVID-19 and Human Rights: We Are All in This Together. https://www.un.org/en/un-coronavirus-communications-team/we-are-all-together-human-rights-and-covid19-response-and (28 July 2020).

[25] UNITED NATIONS (UN 2020b), Disease Pandemics and the Freedom of Opinion and Expression. UN Doc. A/HRC/44/49. https://www.ohchr.org/EN/Issues/FreedomOpinion/Pages/ReportDiseasePandemic s.aspx ,(17 August 2020).

[26] World Health Organization (WHO 2020a), Coronavirus Disease (COVID-19) Dashboard, https://perma. cc/567D-J854

[27] World Health Organization (WHO 2020b), Working Together to Tackle the 'Infodemic.' https://www.euro. who.int/en/health-topics/Health-systems/pages/news/news/2020/6/working-together-to-tackle-theinfodemic (28 July 2020).

\section{AUTHOR'S BIOGRAPHY}

Minas Samatas, is Professor Emeritus of Sociology at the University of Crete, Greece. He has published on surveillance in Greece and other pertinent surveillance issues.

Citation: Minas Samatas. "Biopolitical Surveillance Vs COVID-19 in Authoritarian and Democratic States and Its Possible Post-Pandemic Implications " International Journal of Humanities Social Sciences and Education (IJHSSE), vol 8, no. 4, 2021, pp. 42-51. doi: https://doi.org/10.20431/2349-0381.0804006.

Copyright: (C) 2021 Authors. This is an open-access article distributed under the terms of the Creative Commons Attribution License, which permits unrestricted use, distribution, and reproduction in any medium, provided the original author and source are credited. 